\title{
A MANOMETRIA ANORRETAL (MÉtOdO dO BALÄO) NO DIAGNÓSTICO DIFERENCIAL DA DOENÇA DE HIRSCHSPRUNG
}

\author{
Mauro Batista de Morais*, Vera lucia Sdepanian, Soraia tahan, Soraya Goshima, Ana Cristina Fontenele Soares, Maria Eugênia Farias Almeida \\ Motta, Ulysses Fagundes Neto \\ Trabalho realizado na disciplina de Gastroenterologia Pediátrica - Departamento de Pediatria da Universidade Federal de São Paulo - Escola \\ Paulista de Medicina - São Paulo - SP.
}

\begin{abstract}
RESUMO
OвJEtIVo. Apresentar os resultados dos exames de manometria anorretal realizados com um aparelho brasileiro computadorizado, que utiliza o método do balão para a caracterização do reflexo inibitório reto anal na investigação diagnóstica da doença de Hirschsprung, em crianças com constipação crônica.

Métodos. Foram analisados os resultados de 372 manometrias anorretais realizadas consecutivamente em pacientes com constipação crônica. O equipamento utilizado (Proctosystem-Viotti) possui dois canais utilizados para registar as pressões do esfíncter anal e da ampola retal por meio de balóes conectados por sonda aos transdutores de pressão. Os valores pressóricos são avaliados em computador utilizando software específico. A ausência do reflexo inibitório reto-anal foi considerada como sugestiva de doença de Hirschsprung, sendo o diagnóstico confirmado pelos métodos tradicionais.

RESULTADOs. Em I4 (3,8\%) dos 372 exames não foi caracterizado o reflexo inibitório reto-anal. Em nove destes 14 pacientes o diagnóstico de doença de Hirschsprung foi confirmado pela caracterização de ausência de células ganglionares na avaliação anatomopatológica. Nos outros cinco pacientes não foi realizada a biópsia retal em função da evolução clínica satisfatória com o tratamento clínico da constipação, sendo que em quatro a presença de reflexo inibitório reto-anal foi caracterizada por exame de manometria anorretal realizado posteriormente.

Conclusão. $O$ equipamento utilizado foi apropriado para a realização da manometria anorretal permitindo descartar 0 diagnóstico de doença de Hirschsprung em $86,5 \%$ dos pacientes. Foi identificado um pequeno grupo de pacientes nos quais, em mais da metade, o diagnóstico definitivo de doença de Hirschsprung foi estabelecido.
\end{abstract}

*Correspondência:

Rua Pedro de Toledo, 441,

São Paulo, SP, Cep: 04039 .

UNITERMOs: Constipação. Manometria anorretal. Doença de Hirschsprung. Criança.

031

\section{INTRODUÇÃO}

Constipação crônica constitui a principal queixa em $20 \%$ a $25 \%$ das consultas realizadas em ambulatórios de gastroenterologia pediátrica e em $3 \%$ das consultas de pediatria geral ${ }^{1-4}$. Esta elevada demanda é gerada pelos pacientes que desenvolvem complicações da constipação crônica, dentro do grande universo de crianças que apresentam constipação na comunidade que, segundo estudos realizados no Brasil, ocorre em cerca de $20 \%$ a $30 \%$ da população pediátrica ${ }^{3}$.

A maioria dos pacientes apresenta constipação crônica funcional, que resulta da interação de vários fatores etiológicos, entre os quais dieta pobre em fibra alimentar, episódios de evacuação dolorosa desencadeando círculo vicioso com comportamento de retenção fecal, além de fatores constitucionais e anormalidades na motilidade intestinal ${ }^{1-5}$. Entre os vários diagnósticos diferenciais que devem ser considerados na avaliação da criança com constipação crônica, inclui-se a doença de Hirschsprung ou megacólon congênito $0^{1-4}$. $O$ tratamento da doença de Hirschsprung é cirúrgico e parcela dos pacientes apresenta quadro clínico semelhante ao de pacientes com constipação crônica funcional ${ }^{1-4}$. Na manometria anorretal, os pacientes com doença de Hirschsprung não apresentam o reflexo de relaxamento do esfíncter anal secundário à inflação do balão locado na ampola retal (reflexo inibitório reto-anal) ${ }^{6}$. 0 diagnóstico da doença de Hirschsprung deve ser confirmado pela confirmação anatomopatológica da ausência das células ganglionares dos plexos de Meissner e Auerbach ${ }^{6-8}$.

O objetivo deste estudo éapresentar os resultados dos exames de manometria anorretal realizados com um aparelho brasileiro computadorizado, que utiliza o método do balão para a caracterização do reflexo inibitório reto-anal, na investigação diagnóstica da doença de Hirschsprung em crianças com constipação crônica.

\section{Métodos}

Para avaliar a efetividade da manometria anorretal, foram incluídos no estudo todos os resultados deste tipo de exame realizado consecutivamente na Disciplina de Gastroenterologia Pediátrica da Universidade Federal de São Paulo - Escola Paulista de Medicina (UNIFESP-EPM), no período de novembro de 1999 a novembro de 2003. Neste período, 39l pacientes foram encaminhados para realização do exame, porém 19 pacientes não permitiram a sua realização. Assim, foram analisados 372 
Morals MB et al.

exames realizados em crianças com idade entre 53 dias e 15 anos e 5 meses (média igual a 65 meses). Quando o paciente realizou mais de um exame, na avaliação inicial da efetividade foi considerado apenas o resultado da primeira manometria anorretal.

Dos 372 pacientes, 233 estavam em acompanhamento no Ambulatório de Distúrbios da Motilidade da Disciplina de Gastroenterologia Pediátrica da UNIFESP-EPM. Os outros I 39 pacientes vieram referendados de outros serviços. A maior parte dos médicos que encaminharam esses pacientes havia realizado treinamento de gastroenterologia pediátrica em nosso serviço, o que facilitou a obtenção de informações a respeito da evolução clínica destes pacientes.

Pelo menos duas horas antes de se iniciar a manometria anorretal, foi realizado enema para esvaziamento retal com solução de sorbitol $\left(\right.$ Minilaxa ${ }^{\circledR}$ ) para lactentes e de fosfato hipertônico (FleetEnema ${ }^{\circledR}$ ) para crianças maiores. Foi utilizado aparelho de manometria anorretal (Proctosystem-Viotti, São Paulo, SP) com dois canais para registro dos valores pressóricos captados pelo método do balão. Esse aparelho é conectado a um computador que mostra os gráficos dos valores pressóricos de cada um dos canais durante a realização do exame, por meio de um software específico. Um dos canais mede a pressão do esfíncter anal, sendo o balão (diâmetro aproximado de $0,5 \mathrm{~cm}$ ) e 0 correspondente cateter preenchidos com água. $O$ segundo canal é conectado ao balão que será colocado na ampola retal e que, inicialmente, permanece vazio. Antes da colocação da sonda, o aparelho era calibrado. A sonda com duas vias era introduzida na ampola retal após lubrificação anal com pomada anestésica. A seguir, o balão destinado para mensurar a pressão anal era posicionado no esfíncter anal. Aguardava-se, quando necessário, a tranqüilização do paciente e a estabilização da pressão anal de repouso para iniciar a pesquisa do reflexo inibitório reto-anal. Não foi utilizado nenhum tipo de sedativo durante a realização dos exames. Procedeu-se, então, a pesquisa do reflexo inibitório reto-anal com a inflação do balão retal com I0, 20, 30,40, 50 e $60 \mathrm{ml}$ de ar. Cada uma das inflações do balão retal foi realizada sempre quando o traçado mostrava pressão esfincteriana estabilizada no valor da pressão anal de repouso. A presença de reflexo inibitório reto-anal foi caracterizada quando ocorreu redução da pressão anal maior ou igual a $5 \mathrm{mmHg}$ em relação ao momento de inflação do balão retal ${ }^{4}$. Valorizou-se, também, a presença do traçado típico do reflexo no gráfico. Quando não se caracterizou a presença do reflexo inibitório reto-anal durante o exame, foi realizado toque retal. Nos pacientes nos quais se evidenciou a presença de fezes na ampola retal, foi realizado enema para esvaziamento do reto antes de prosseguir o exame. Persistindo a ausência do reflexo inibitório reto-anal com a inflação de $60 \mathrm{ml}$ de ar, o volume foi aumentado a cada $20 \mathrm{ml}$, até o volume máximo de $100 \mathrm{ml}$ nos pacientes na faixa etária escolar. Considerou-se como exame inconclusivo quando não foi possível definir a presença ou ausência de reflexo, ou devido a traçado atípico ou devido a não colaboração do paciente.

Os pacientes que apresentaram ausência de reflexo inibitório reto-anal realizaram avaliação complementar de acordo com a conduta de cada médico responsável pela solicitação da manometria anorretal, quando o paciente não se encontrava em acompanhamento em nosso ambulatório. Esses médicos foram contatados posteriormente para obtenção de informações relativas ao diagnóstico definitivo e evolução
Figura I - Manometria anorretal pelo método do balão em paciente com constipação crônica funcional, em que se observa o reflexo inibitório retoanal (relaxamento esfincteriano induzido pela inflação do balão retal). $\mathrm{Na}$ parte superior $(\mathrm{PI})$ da figura, está o registro da pressão no esfíncter interno do ânus. Na parte inferior (P2), observa-se o registro da pressão retal, mostrando os momentos exatos de inflação do balão retal com IO (VI), 20 (V2), 30 (V3) e 40 (V4) ml de ar. Notar aumento crescente do relaxamento esfincteriano (PI) com o aumento da insuflação do balão retal (P2)

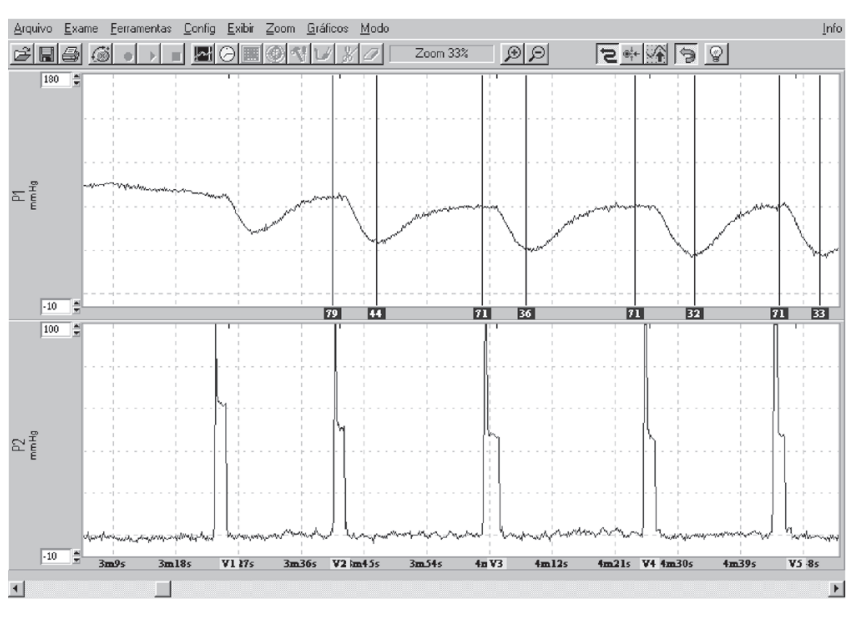

dos pacientes. Considerou-se, ainda, além do resultado da biópsia retal, a evolução clínica após a instituição do tratamento e o resultado de segundo exame de manometria quando o mesmo foi repetido em nosso serviço.

Para os pacientes com exame inconclusivo, foi sugerido aos médicos que solicitassem um segundo exame. Foi calculada a porporção de manometrias anorretais inconclusivas de acordo com as faixas etárias, bem como quanto a época de realização dos exames. Utilizou-se o teste do Qui-quadrado para as comparações e análise estatística.

O projeto do estudo foi analisado e aprovado pelo Comitê de Ética em Pesquisa da Universidade Federal de São Paulo - Hospital São Paulo.

\section{Resultados}

Ausência de reflexo inibitório reto-anal foi observada em I 4(3,8\%) dos 372 exames analisados. Nas Figuras I e 2 são mostrados dois traçados dos exames realizados. A Figura I caracteriza a presença de reflexo inibitório reto-anal, com redução da pressão anal (canal PI) concomitante à inflação do balão retal (canal P2). Observa-se que, com o aumento sucessivo do volume de inflação do balão retal $(\mathrm{VI}=10 \mathrm{ml}$; $\mathrm{V} 2=20 \mathrm{ml} ; \mathrm{V} 3=30 \mathrm{ml} ; \mathrm{V} 4=40 \mathrm{ml}$ ), ocorre aumento da amplitude da redução da pressão anal. Na Figura 2, não se evidencia o relaxamento do esfíncter anal desencadeado pela inflação do balão retal, ou seja, não ocorreu o reflexo inibitório reto-anal.

Em nove dos I 4 pacientes com ausência do reflexo inibitório retoanal foi confirmado o diagnóstico da doença de Hirschsprung pela caracterização anatomopatológica de aganglionose. Os outros cinco pacientes não realizaram biópsia retal, até o presente momento, em 
Figura 2 - Manometria anorretal pelo método do balão em paciente com doença de Hirschsprung de segmento curto. Na parte superior (PI) da figura, está o registro da pressão no esfíncter anal. Na parte inferior (P2), observa-se o registro da pressão retal, mostrando os momentos exatos de inflação do balão retal com 20 (V2), 30 (V3), 40 (V4), 50 (V5) e 60 (V6) ml de ar. Ausência do reflexo de inibitório reto-anal. Observa-se contração do esfíncter externo do ânus na parte superior da figura

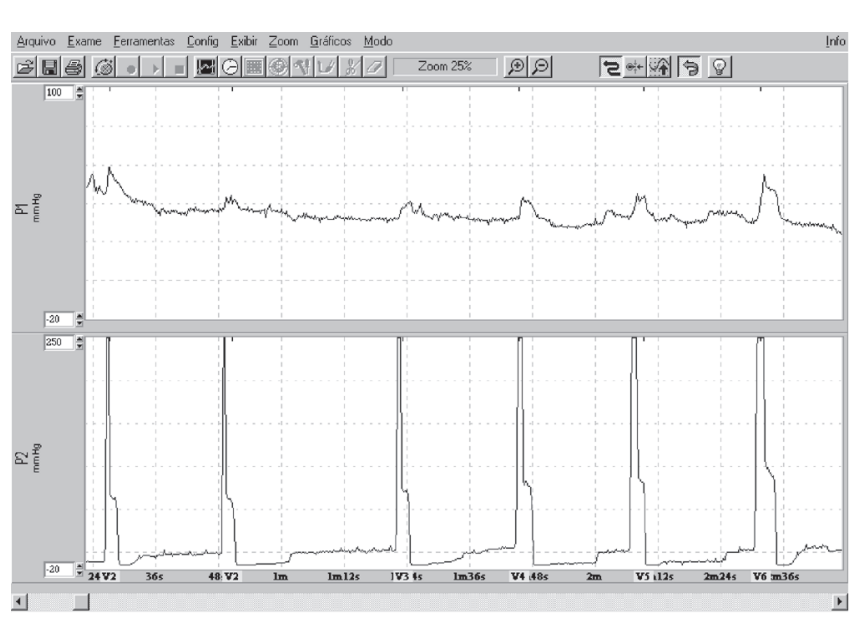

Tabela I - Possibilidade de conclusão das manometrias anorretais de acordo com as faixas etárias estudadas

\begin{tabular}{|c|c|c|c|}
\hline \multirow[t]{2}{*}{ Faixa etária } & \multicolumn{2}{|c|}{ Exame conclusivo } & \multirow[t]{2}{*}{ Total } \\
\hline & Sim & Não & \\
\hline $0-24$ meses & $\overline{55(80,0 \%)}$ & $07(20,0 \%)$ & $62(100,0 \%)$ \\
\hline $24-59$ meses & $101(82,2 \%)$ & $18(17,8 \%)$ & $119(100,0 \%)$ \\
\hline$\geq 59$ meses & $180(93,9 \%)$ & $11(6,1 \%)^{\prime}$ & $191(100,0 \%)$ \\
\hline Total & 336 & 36 & 372 \\
\hline
\end{tabular}

Qui-quadrado $=7,581 \quad(p=0,023)$

Tabela 2 - Possibilidade de conclusão das manometrias anorretais de acordo com a época de realização dos exames

\begin{tabular}{|c|c|c|c|}
\hline \multirow[t]{2}{*}{ Época } & \multicolumn{2}{|c|}{ Exame conclusivo } & \multirow[t]{2}{*}{ Total } \\
\hline & Sim & Não & \\
\hline |999-200| & $137(82,5 \%)$ & $24(17,5 \%)$ & $161(100 \%)$ \\
\hline $2001-2003$ & $199(94,0 \%)$ & $12(6,0 \%)$ & $211(100 \%)$ \\
\hline Total & 336 & 36 & 372 \\
\hline
\end{tabular}

Qui-quadrado $=7,86(p=0,005)$

função de resposta favorável ao tratamento clínico da constipação. Quatro destes cinco pacientes repetiram a manometria anorretal durante o tratamento clínico da constipação e, nesta segunda tentativa, foi possível a demonstração do reflexo inibitório reto-anal. Um destes cinco pacientes não prosseguiu a investigação.

Nos pacientes nos quais se evidenciou a presença de reflexo inibitório reto-anal, não se estabeleceu posteriormente o diagnós- tico de doença de Hirschsprung em nenhum dos 202 pacientes acompanhados em nosso ambulatório, nem nos 120 pacientes encaminhados por médicos de outros serviços.

Em 12 dos 36 pacientes que apresentaram exame inconclusivo foi realizado novo exame, e em 10 foi detectada a presença de reflexo inibitório anal (RIA). Em dois pacientes concluiu-se pela ausência de RIA, mas até o presente momento não realizaram a biópsia para confirmação da aganglionose.

A Tabela I mostra a proporção de exames inconclusivos de acordo com as faixas etárias. A possibilidade de conclusão dos exames foi maior na faixa etária 59 meses $(p=0,023)$, em relação às faixas etárias $\geq$ menores, de 0 a 24 meses e de 24 a 59 meses. A Tabela 2 demonstra que o número de exames inconclusivos foi mais freqüente entre 1999 e 200 I do que entre 2002 e 2003.

\section{Discussão}

Constipação crônica éuma manifestação clínica freqüente na população pediátrica. As crianças com quadros de maior gravidade ou com complicações, especialmente aquelas com escape fecal, são atendidas em ambulatórios especializados. O diagnóstico de constipação crônica funcional deve prevalecer quando a anamnese e o exame físico não revelam indícios de outras causas intestinais ou sistêmicas de constipação. Nessa circunstância, recomenda-se o tratamento convencional da constipação crônica, que inclui esvaziamento do fecaloma, quando necessário, medidas educativas, maior consumo de fibra alimentar e líquidos, além da prescrição de laxante ${ }^{1-4}$. Para os pacientes que não apresentam resposta favorável ao tratamento ou impossibilidade de suspensão dos laxantes é necessária a indicação de procedimentos diagnósticos adicionais. De acordo com a recomendação da Sociedade Norte-Americana de Gastroenterologia Pediátrica, na primeira fase de investigação das crianças com constipação crônica, deve ser descartado - diagnóstico de doença de Hirschsprung com o emprego da manometria anorretal ${ }^{2}$. Em nosso serviço, a mesma conduta vem sendo adotada. Deve ser lembrado que a pesquisa da doença de Hirschsprung é indicada na avaliação inicial dos pacientes que apresentam as seguintes manifestações clínicas de alerta: retardo na eliminação de mecônio, constipação desde o nascimento, déficit de crescimento, distensão abdominal, fezes com forma de fita e eliminação explosiva de fezes durante o toque retal ${ }^{-4}$. No entanto, deve ser mencionado que muitos pacientes com constipação crônica funcional grave apresentam constipação desde o nascimento ${ }^{1-4}$.

A escolha da manometria anorretal como método inicial de avaliação na suspeita de doença de Hirschsprung é um ponto importante a ser considerado, pois, em nosso meio, freqüentemente o enema opaco é o primeiro procedimento diagnóstico indicado. $O$ enema opaco pode contribuir para o diagnóstico das formas clássicas da doença de Hirschsprung, entretanto, nas formas com segmento curto e, principalmente, ultra-curto, esse diagnóstico deve ser descartado pela demonstração do reflexo inibitório reto-anal ${ }^{6-8}$. Outro aspecto a ser mencionado é que o enema opaco provoca mais desconforto para o paciente do que a manometria anorretal, além da necessidade de exposição radiológica.

A manometria anorretal também tem sido indicada na investigação 
Morais MB ET AL.

diagnóstica de recém-nascidos, incluindo pré-termos, com atraso de eliminação de mecônio e sintomas de obstrução intestinal baixa, demonstrando-se na literatura sua utilidade diagnóstica na identificação da doença de Hirschsprung ${ }^{12,20-23}$. Em nossa casuística não tivemos nenhum recém-nascido, porém incluímos lactentes jovens, o menor com 53 dias e vários com menos de 6 meses $(n=15)$, nos quais foi possível demonstrar a presença do reflexo inibitório reto-anal com 0 método da manometria anorretal utilizando o método do balão.

Existem várias técnicas para realizar a manometria anorretal e os aparelhos de perfusão com múltiplos canais para captação de pressão são os mais utilizados na Europa e na América do Norte. No presente estudo, foi utilizado um aparelho nacional, com menor custo, que se baseia no método do balão para mensuração da pressão, com dois canais, um utilizado para registro da pressão esfincteriana e outro para assinalar os momentos de inflação do balão retal para desencadear o reflexo inibitório reto-anal. Em 322 (86,5\%) pacientes avaliados nesta série foi possível descartar o diagnóstico da doença de Hirschsprung no primeiro exame de manometria, pela caracterização do reflexo inibitório reto-anal, permitindo maior segurança no acompanhamento e no tratamento desses pacientes com constipação crônica funcional, sem que fosse necessária a complementação diagnóstica por enema opaco e/ou biópsia retal. Nos pacientes deste estudo, no segundo exame de manometria, permitiram a demonstração do reflexo quatro pacientes nos quais não se havia identificado reflexo no primeiro exame e em 10 dos 22 nos quais a manometria foi inconclusiva na primeira tentativa. Se estes resultados forem considerados em conjunto, manometria anorretal permitiu afastar o diagnóstico da doença de Hirschsprung em $90 \%$ dos casos avaliados.

Para a manometria anorretal, como para qualquer método diagnóstico, um aspecto importante a ser considerado é a sensibilidade e a especificidade do exame como indicação da eficácia diagnóstica. Para tal, é fundamental a definição do padrão ouro para o diagnóstico de certeza da doença em questão. No caso da doença de Hirschsprung, o padrão ouro é a caracterização de ausência das células ganglionares dos plexos de Meissner e Auerbach em fragmentos de biópsia retal cirúrgica corada pela hematoxilina e eosina ${ }^{4-6}$. No entanto, com exceção de um estudo', em todos os outros analisados na literatura a respeito do desempenho diagnóstico da manometria anorretal ${ }^{10-18}$, a biópsia retal profunda só foi realizada quando não se demonstrou a presença do reflexo inibitório reto-anal. Em nosso estudo, conduta idêntica foi adotada. Assim, em 9 dos 14 pacientes com ausência do reflexo inibitório reto-anal, foi confirmado o diagnóstico de doença de Hirschsprung por meio de biópsia retal profunda. Por outro lado, os outros cinco pacientes nos quais não se identificou o reflexo inibitório reto-anal não realizaram biópsia retal até o presente momento em função da evolução satisfatória com o tratamento clínico da constipação, ressaltando-se que em quatro deles a caracterização do reflexo inibitório reto-anal foi possível em manometria anorretal realizada posteriormente. Esse número de resultados falso-positivos, aparentemente elevado, deve ser analisado frente aos 322 casos nos quais se caracterizou o reflexo inibitório reto-anal no primeiro exame, o que atesta a aplicabilidade clínica da manometria anorretal, especialmente por evitar a realização de enema opaco e biópsia retal profunda. A aplicabilidade clínica fundamenta-se, ainda, na grande desproporção entre as prevalências de doença de Hirschsprung e constipação funcional como causas de constipação crônica em Pediatria. Assim, nossos resultados indicam que a manometria anorretal foi adequada, utilizando o método do balão, no que se refere ao seu desempenho diagnóstico em condições usuais de operação. Nossos resultados são semelhantes aos encontrados em estudo realizado no Brasil com o emprego de aparelho estrangeiro de manometria de perfusão, no qual se diagnosticou doença de Hirschsprung em oito $(61,5 \%)$ dos pacientes nos quais não se identificou reflexo inibitório reto-anal ${ }^{18}$. Em nosso estudo, doença de Hirschsprung foi diagnosticada em nove $(64,3 \%)$ dos pacientes que não apresentaram reflexo inibitório reto-anal, considerando o primeiro exame.

$O$ equipamento e o software produzidos no Brasil permitiram a realização do exame com performance adequada, conforme ilustrado nos traçados apresentados nas Figuras I e 2. Os resultados falso positivos (ausência de reflexo inibitório reto-anal em crianças com constipação crônica funcional) podem ser decorrentes da presença de megarreto, que pode requerer inflação de volumes maiores no balão retal para desencadear o reflexo inibitório reto-anal, ou ainda pela presença de fezes impactadas no reto, razão pela qual é importante realizar toque retal quando não se obtém o reflexo inibitório reto-anal durante a manometria anorretal, devendo-se, então, proceder a limpeza colônica para prosseguir o exame. Por sua vez, resultados falso-negativos (presença de reflexo inibitório reto-anal em paciente com doença de Hirschsprung), teoricamente, podem ocorrer por problemas técnicos durante a realização do exame que podem ser reconhecidos levando em consideração as características do traçado obtido, além da diminuição da pressão esfincteriana. Na nossa prática, observamos que resultados inconclusivos decorrem principalmente da pouca ou não colaboração do paciente ou devido a traçado duvidoso ou atípico. Crianças pequenas ou muito traumatizadas com lavagens intestinais freqüentes e ainda aquelas que apresentam comportamento de retenção importante são menos colaborativas na realização do exame, dificultando a sua conclusão. Nossos resultados demonstraram que a possibilidade de conclusão do exame foi maior nas crianças com idade maior que 59 meses (Tabela I). A possibilidade de conclusão do exame também foi mais freqüente no período de 2002 a 2003 em relação ao período inicial de 1999 a 2001 . Acreditamos que este fato decorra do acúmulo de maior experiência pela equipe que realiza os exames com o passar dos anos, refletindo em maior efetividade da manometria no período mais recente.

Pelo exposto, em concordância com o recomendado pela Sociedade Norte-Americana de Gastroenterologia Pediátrica e Nutrição ${ }^{(2)}$ e por outros autores ${ }^{4,17}$, é possível reafirmar que a manometria anorretal deve ser o primeiro exame a ser indicado na avaliação da doença de Hirschsprung em crianças com constipação crônica e naquelas que apresentam as manifestações clínicas de alarme sugestivas de aganglionose colônica para descartar o diagnóstico de doença de Hirschspung.

\section{Conclusão}

O equipamento utilizado foi apropriado para a realização da manometria anorretal em crianças com constipação crônica, permitindo descartar o diagnóstico de doença de Hirschsprung em 86,5\% 
dos pacientes avaliados, considerando o primeiro exame realizado. Foi possível, também, identificar um pequeno grupo de pacientes nos quais, em mais da metade, o diagnóstico definitivo de doença de Hirschsprung foi estabelecido.

\section{Conflito de interesse: não há.}

\section{SUMMARY}

Effectiveness OF anorectal manometry using the BALLOON METHOD TO IDENTIFY THE INHIBITORY RECTO-ANAL REFLEX FOR DIAGNOSIS OF HIRSCHSPRUNG'S DISEASE

OBJECTIVE. to evaluate results of anorectal manometry performed with equipment made in Brazil for the screening of Hirschsprung's disease in children with chronic constipation.

METHODS. Results of 372 anorectal manometries performed consecutively in children with chronic constipation were evaluated. The equipment (Proctosystem Viotti) has two channels for pressure registration by the balloon method and is connected to a computer using specific software. Absence of the inhibitory recto-anal reflex was considered suggestive of Hirschsprung's disease and diagnosis was confirmed by traditional diagnostic methods.

RESULTS. Absence of the inhibitory recto-anal reflex was found in 14 (3.8\%) of the 372 anorectal manometry examinations. Diagnosis of Hirschsprung disease was confirmed in 9 out of 14 patients by characterization of aganglionosis upon rectal biopsy. In the other 5 patients, rectal biopsy was not performed in view of a satisfactory evolution with the clinical treatment for constipation. In 4 out of the 5 patients the inhibitory recto-anal reflex was demonstrated with a second anorectal manometry examination.

Conclusions. The equipment used for anorectal manometry presented a satisfactory performance. Diagnosis of Hirschsprung disease was discarded in $86.5 \%$ of the patients with chronic constipation because the inhibitory recto-anal reflex was detected. Manometric evaluation also made possible the identification of a small group of patients in which more than half had Hirschsprung's disease. [Rev Assoc Med Bras 2005; 5 I (6): 3|3-7]

KeY wORDS: Constipation. Anorectal manometry. Hirschsprung's disease. Children.

\section{REFERÊNCIAS}

I. Loening-Baucke V. Encopresis and soiling. Pediatr Clin North Am 1996:43:279-298.

2. Baker SS, Liptak GS, Colletti RB, Croffie JM, Di Lorenzo C, EctorW, et al. Constipation in infants and children: Evaluation and treatment. A medical position statement of the North American Society for Pediatric Gastroenterology and Nutrition. J Pediatr Gastroenterol Nutr 1999; 29:6I2-26.

3. Morais MB, Maffei HVL. Constipação intestinal. J Pediatr (Rio de J) 2000; 76:SI47-S56

4. Di Lorenzo C. Pediatric anorectal disorders. Gastroenterol Clin North Am 200I;30:269-87.
5. Morais MB, Vítolo MR, Aguirre ANC, Fagundes-Neto U. Measurement of low dietary fiber intake as a risk factor for chronic constipation in children. J Pediat Gastroenterol Nutr 1999; 29:132-5.

6. Loening-Baucke V. Anorectal manometry and biofeedback training. In: Hyman PE, Di Lorenzo C, editors. Pediatric gastrointestinal motility disorders. New York: Academy Professional; 1994. p.23I-52.

7. Kirschner BS. Hirschsprung's disease. In:Walker A, Durie Pr, Hamilton JR, Walker-Smith JA, Watkins JB. Pediatric gastrointestinal disease. 3rd ed. St. Louis: Mosby; 2000. p.844-8.

8. Méndez V. Doença de Hirschsprung. In: Ferreira CT, Carvalho E, Silva LR, editores. Gastroenterologia e hepatologia em pediatria. Rio de Janeiro: Medsi; 2003. p.237-52.

9. Low PS, Quak SH, Prabhakaran K, Joseph VT, Chiang GS, Aiyathurai EJ. Accuracy of anorectal manometry in the diagnosis of Hirschsprung's disease. J Pediatr Gastroenterol Nutr 1989;9:342-6.

10. Loening-Baucke VA. Anorectal manometry: experience with strain gauge pressure transducers for the diagnosis of Hirschsprung's disease. J Pediatr Surg 1983; 18:595-600.

II. Lanfranchi GA, Bazzocchi G, Federici S, Brignola C, Campieri M, Rossi F, et al. Anorectal manometry in the diagnosis of Hirschsprung's diseasecomparison with clinical and radiological criteria. Am J Gastroenterol 1984;79:270-5

12. Loening-Baucke V, Pringle KC, Ekwo EE. Anorectal manometry for the exclusion of Hirschsprung's disease in neonates. J Pediatr Gastroenterol Nutr 1 985;4:596-603.

13. Iwai N, Yanagihara J, Tokiwa K, Deguchi E, Perdzynski W, Takahashi T. Reliability of anorectal manometry in the diagnosis of Hirschsprung's disease. Z Kinderchir 1988;43:405-7.

14. Nagasaki A, Sumitomo K, Shono T, Ikeda K. Diagnosis of Hirschsprung's disease by anorectal manometry. Prog Pediatr Surg 1989;24:40-8.

I5. Gil Vernet JM, Casasa JM, Lloret J, Marhuenda C, Broto J, Boix Ochoa J. Manometria anorrectal en la enfermedad de Hirschsprung. Cir Pediatr 1992;5:135-40.

16. Morera C, Rodriguez M, Prados J, Jaen D. Manometría anorectal: experiencia de 5 años. GEN 1995;49:2 I 2-7.

17. Osatakul S, Patrapinyokul S, Osatakul N. The diagnostic value of anorectal manometry as a screening test for Hirschsprung's disease. J Med Assoc Thai 1999;82: I 100-5.

18. Costa-Pinto EAL, Bustorff-Silva JM, Fukushima E. Papel da manometria anorretal no diagnóstico diferencial da constipação em crianças. J Pediatr (Rio de J) 2000;76:227-32.

19. Yokoyama J, Kuroda T, Matsufugi H, Hirobe S, Hara S, Katsumata K. Problems in diagnosis of Hirschsprung's disease by anorectal manometry. Prog Pediatr Surg 1989;24:49-58.

20. Loening-Baucke $V$, Kimura K. Failure to pass meconium: diagnosing neonatal intestinal obstruction. Am Fam Physician 1999; 60:2043-50.

2I. Núñez R, Vargas I, Cabrera R, Espinosa J, Blesa E. Manometría anorretal em el recíen nacido. Cir Pediatr 1993;6: I I I-3.

22. López-Alonso M, Ribas J, Hernández A, Anguita FA, Gómez de Terrenos I, Martínez-Caro A. Efficiency of the anorectal manometry for the diagnosis of Hirschsprung's disease en the newborn period. Eur J Pediatr Surg 1995;5:160-3.

23. Emir H, Akman M, Sarimurat N, Kiliç N, Erdogan E, Soylet Y. Anorectal manometry during the neonatal period: its specificity in the diagnosis of Hirschsprung's disease. Eur J Pediatr Surg 1999;9: I0I.

24. Loening-Baucke V. Constipation in children. Curr Opin Pediatr | 994;6:556-61.

Artigo recebido: 09/08/04

Aceito para publicação: 03/02/05 